\title{
548 - Cognitive aging and cognitive reserve
}

\author{
Alena Sidenkova
}

Ural State Medical University, Yekaterinburg, Russian Federation

Introduction. The aging processes are accelerating in all regions of the world. The involvement of older people in production and social processes determines the need to maintain a high level of social and psychological adaptation, despite the progressive pathology of the brain caused by its aging. This increases the relevance of research related to the study of biological reserves of the brain and psychological and social mechanisms of human adaptation in late adulthood. The risk of developing cognitive disorders is not fatal. According to some observations, even in the hippocampal type of UKR, despite the content of amyloid in the brain, the functional and social activity of the elderly remains high. Prospective studies show that people with high cognitive reserve have a lower risk of developing dementia. Cognitive reserve is the brain's resistance to damage. Cognitive reserve is the ability of the brain to cope with the consequences of damage caused by external influences, brain stroke, chronic brain ischemia, neurodegenerative diseases, and age-related changes. Cognitive reserve is the brain's ability to functionally compensate for and minimize clinical manifestations of cognitive impairment. The mechanisms of cognitive reserve in normal and Alzheimer's disease are different. In healthy older adults, a higher cognitive reserve correlates with larger brain sizes and effective strategies for performing cognitive tasks. In the early stages of Alzheimer's disease and Alzheimer's disease, the size of the brain decreases. But high brain activity helps preserve cognitive resources. Excessive brain activity in dementia is a compensatory mechanism. This is confirmed by the results of functional magnetic resonance imaging of the brain. Of course, the degree of brain atrophy is a predisposing factor for dementia, but it is not a mandatory factor for cognitive decline. So, the symptoms of dementia do not appear until you have crossed the critical border of damage to the brain substance. Progressive brain atrophy underlies the clinical manifestations of dementia in neurodegenerative diseases, but the correlation between the degree of brain damage and cognitive impairment is not linear.

Research materials and methods. An observational 10-year longitudinal study was conducted. In 2006, moderate cognitive impairment was found in 66 patients. The group of patients included 49 women and 49 men. Their average age in 2006 was $59.3 \pm 5.2$ years. In 2006, the severity of cognitive decline was $26.2 \pm 1.9$ points on the MMSE scale. This corresponds to indicators of moderate cognitive impairment. Research methods: clinical and psychopathological, psychometric, statistical. Questionnaire "Loss and acquisition of personal resources" (N. Vodopyanova, M. Stein), MMSE scale.

Research result. In 2006, amyloid was detected in the spinal fluid of all patients selected for the study group. If a patient developed dementia, they were given specific therapy. The dynamics of cognitive functions in patients was different. Mild dementia was formed in $53 \%$ of patients. Moderate dementia was formed in $10.6 \%$ of patients. Moderate cognitive impairment (pre-dementia) persisted in $36.4 \%$ of patients. Hereditary burden of dementia in patients with moderate dementia was detected 2 times more often. Back in 2006, we identified the leading sensory systems of patients. The master sensor system determines the modality of the main information flow. This is the most important part of the

information that a person interacts with. This is the basis of interaction with reality. This is the basis of cognitive functions. Correlations of age-specific lesions of the corresponding sensory systems with the severity of cognitive decline were found in patients with the corresponding sensory modality (Spearman's Correlation Coefficient- $r, p<0.05$ ): presbyacoussis - auditory $r=0.667$, presbyopia-visual $r=0.705$. The influence of psychosocial factors on the condition of patients was studied. In dementia, significant history of psychotrauma was found in $35.7 \%$. Moderate stress was detected in the group of patients with moderate cognitive impairment in $33.3 \%$. Moderate stress was detected in the group of patients with dementia in $83.3 \%$. Stress of loss of life meaning was detected more often in patients with dementia $76.7 \%$. It is important not only what stresses a person endures, but how they can cope with them. Dementia patients were statistically more likely to have unproductive coping strategies that did not help them cope adequately with stress. 
Conclusions. The concept of cognitive reserve suggests possible causes of heterogeneity in the dynamics of cognitive decline in the initial stages of atrophic-degenerative brain diseases: biological causes and psychosocial causes. The concept of cognitive reserve helps to study and develop individual programs for the prevention of severe cognitive disorders.

\section{9 - Psychosocial factors in the formation of non-cognitive symptoms of dementia Alena Sidenkova \\ Ural State Medical University, Yekaterinburg, Russia}

Background: The growing prevalence of severe cognitive impairment in populations, the involvement of a significant number of people of working age in the medical, economic, psychological and social problems associated with late dementia, the insufficiency and inconsistency of information about the mechanisms of formation of these disorders actualize a comprehensive medical and social study of dementia.

The goal is to study the psychosocial mechanisms of the formation of clinical, functional disorders in dementia, to develop comprehensive medical and psychosocial programs to help patients with dementia and those involved in caring for them, based on the proposals of the psychosocial model of dementia.

Methods: A selective observational comparative dynamic study of 315 people with Alzheimer's dementia and 214 people who care for the patients was carried out. The study used clinical, clinical, psychopathological, neuropsychological, psychometric, sociometric, and statistical research methods.

Results: An increase in the severity of dementia contributes to a significant $(p<0.05)$ increase in the number of patients in a dependent position in the family. The appearance of psychotic (painful ideas $[r=$ $0.589]$ ), behavioral (agitation / aggression [ $r=0.654]$ ), affective (anxiety [ $r=0.536]$, unstable mood / irritability $[r=0.581]$ ) symptoms of dementia contribute to family role changes structure and increase interpersonal distance in the dyad "caretaker - patient." Decreased functional activity of the patient $(r=$ 0.758), development of behavioral disorders (aberrant behavior $[r=0.675]$, agitation / aggression $[r=$ 0.713], impaired night behavior [ $r=0.597]$ ), affective symptoms (anxiety [ $r=0.685]$ ) contribute to aggravation of the distress of the caregiver. Those who provide unprofessional care for dementia patients in a statistically significant $(p<0.05)$ majority of cases have a high level of emotional involvement in the care process. Changes in family-role and social parameters, a high level of "expressed" emotions of caregivers have an adverse effect on the development of psychotic $(r=0.618)$, affective $(r=0.701)$, behavioral $(r=0.837)$ dementia disorders. The degree of adherence to antidementia therapy by the caregiver is one of the important factors determining the amount of care received by the patient $(r=0.698)$. Agitation / aggression $(r=0.761)$, anxiety $(r=0.562)$, sleep disturbances $(r=0.521)$ contribute to increased compliance. The low satisfaction of the caregiver with premorbid $(r=0.698)$ and current $(r=0.653)$ relationships with the patient leads to a decrease in the compliance of the caregiver.

Conclusion: It was revealed that the formation of cognitive impairment is caused by biological factors, their severity depends on the severity of dementia. The mechanism of psychopathological symptoms, functional disorders is heterogeneous, depending on the biological causes and psychosocial conditions of functioning of patients. 